\title{
The Publication Rates of Pediatric Rheumatology Abstracts Presented in European League Against Rheumatism 2009 Congress
}

\author{
Özge Altuğ GÜCENMEZ, ${ }^{1}$ Deniz BAYRAKTAR, ${ }^{2}$ Umut Ziya KOÇAK, ${ }^{2}$ \\ Balahan MAKAY, ${ }^{1}$ Şevket Erbil ÜNSAL ${ }^{1}$ \\ ${ }^{1}$ Department of Pediatric Rheumatology, Medical Faculty of Dokuz Eylül University, İzmir, Turkey \\ ${ }^{2}$ School of Physical Therapy and Rehabilitation, Dokuz Eylül University, Izmir, Turkey
}

\begin{abstract}
Objectives: This study aims to evaluate the publication rates and features of the abstracts related to pediatric rheumatology presented in European League Against Rheumatism 2009 congress.

Methods: A systematic search was performed to find full-text publications of abstracts related to pediatric rheumatology in European League against Rheumatism 2009 congress. Full-text publication rate, the elapsed time between presentation and full-text publication, type of the disease in the studies, distribution of abstracts and full-texts according to countries, journals, and presentation types were investigated.

Results: Totally 220 abstracts were detected related to pediatric rheumatology. Twenty-two of them had only the title, authors' names, and institution of origin, but no abstract. Therefore, a total number of 198 abstracts were evaluated. Eighty-six (43.4\%) abstracts were found to be accepted as full-text articles. The elapsed time between presentation and full text publication was median 19 months (range 0 to 64 months). While 12 orally presented abstracts (34\%) became full-text articles, this rate was $45 \%$ ( 74 abstracts) for poster presentations. There was no significant difference in the elapsed time to reach full-text publication and impact factors between presentation types ( $p=0.832$ and $p=0.053$, respectively).

Conclusion: The full-text publication rates were within similar ranges when compared to other reports in rheumatology field. It seems that even though European League against Rheumatism is a general rheumatology congress, it takes an important place in pediatric rheumatology field as well.

Keywords: Pediatric rheumatology; publication rates; rheumatology congress.
\end{abstract}

Pediatric rheumatology subspecialty was born in the $19^{\text {th }}$ century due to the lack of pediatricians' ability to cover the whole field of the pediatrics. The first attention was commonly paid on rheumatic fever in the early stages of its development, but then other rheumatic diseases of childhood attracted attention. Pediatric rheumatology is considered as one of the latest and the least populated subspecialty in the pediatrics. However, its development is increasing day by day both clinically and scientifically. ${ }^{1}$

Scientific meetings are intended to gather clinicians, researchers and even patients together, allowing the sharing of ideas to contribute in professional network. Also, meetings enable researchers to share their works with their peers and experts from the field. Oral and poster presentations are the most common forms in this regard. Abstracts can be described as the first fruits which a researcher gets, but unless they become full-text publications, their usefulness will be limited. Even though an abstract includes the most important data, only a limited group (the participants of the congress or the ones who can reach to the abstract book) would be aware of it without a full-text publication. Also, it was advocated that abstract to full-text rate shows the importance of the congress in the related field. ${ }^{2}$ 
Although the publication rates of a pediatric rheumatology congress were reported, ${ }^{3}$ to our knowledge, no research was conducted on full-text publication rates of pediatric rheumatology abstracts which were presented in a general and leading rheumatology congress such as the Annual European Congress of Rheumatology of the European League Against Rheumatism (EULAR). Therefore, in this study, we aimed to evaluate the publication rates and features of the abstracts related to pediatric rheumatology presented in EULAR 2009 congress.

\section{METHODS}

We conducted the study between November 2014 and January 2015 at the Department of Pediatric Rheumatology, Medical Faculty of Dokuz Eylül University. We performed abstract data extraction, systematic search for full-text publications, and full-text publication data extraction according to a method which was modified from the study by Smith et al. ${ }^{4}$ An ethical approval was not required, since it was a screening method which did not include any patient nor author data.

The presented abstracts in EULAR 2009 were archived in The European League Against Rheumatism Abstracts 2 View ${ }^{\mathrm{TM}}$ site. ${ }^{5}$ We screened this site and included all the abstracts related to pediatric rheumatology in the study. We chose the year 2009, because it was advocated that five-years was a suitable time to show the full-text publication rates of the previously presented abstracts. ${ }^{4}$

Two independent researchers entered each abstract into a database. We recorded the title of the abstract, name of authors, type of presentation, and country of the origin. When we encountered a multicenter study abstract, we determined the country of the origin according to the country of the first author. We resolved the discrepancies in recording basic data from presented abstracts by a consensus with a third researcher. We based the consensus on the predominance of the decisions. In such cases, we accepted the decision of the third researcher as the final decision when two of the three researchers were in agreement.

Two researchers searched the titles and authors of the abstracts in Pubmed, ${ }^{6}$ Web of Science, ${ }^{7}$ and Google Scholar ${ }^{8}$ databases. We resolved the discrepancies in search results by a consensus with a third researcher as aforementioned. We sought potential articles firstly by searching for the title and first author of the abstract. If we found no corresponding article, we repeated the search up to five times in each database using alternative authors and key words. We retrieved all the possible full-text publications and verified the concordance between the information contained in the presented abstract and full-text publication to ensure that they represent the same body of work.

Two independent researchers reviewed each full-text publication and recorded the following basic data: authors' names, publication title, journal name, and the date of publication. We subsequently calculated the time of full-text publication in months as being the time between the date of abstract presentation and the date of full-text publication (not the e-pub time). We resolved the discrepancies in recording basic data from full-text publications by a consensus with a third investigator. In such cases, we accepted the decision of the third investigator as the final decision. We obtained the impact factors of the journals by searching the Institute of Scientific Information's Journal Citation Reports Science Edition for 2014, due to the absence of the 2015 report. $^{9}$

\section{Statistical analysis}

We performed the statistical analyses using the IBM SPSS version 20.0 software (IBM Corporation, Armonk, NY, USA). We summarized data as median and minimum-maximum range or percentages. We used the Mann-Whitney $\mathrm{U}$ test to determine the differences between presentation types (oral vs poster) and set the level of significance at $p<0.05$.

\section{RESULTS}

We detected a total of 220 abstracts related to pediatric rheumatology. Twenty-two of them had only the title, authors' names, and institution of origin, but no body text in the Abstracts2 $\mathrm{View}^{\mathrm{TM}}$ site. Therefore, we conducted the below analysis over 198 abstracts. We found 86 (43.4\%) abstracts as full-text articles. 
Table 1. Subcategories of presented abstracts of European League Against Rheumatism 2009 Congress

\begin{tabular}{|c|c|c|c|}
\hline & Abstract & Full-text & Abstract to full-text rate \\
\hline & $\mathrm{n}$ & $\mathrm{n}$ & $\%$ \\
\hline Advances in pediatric rheumatology & 6 & 4 & 67 \\
\hline Presentation of abstracts that were selected for the PReS & & & \\
\hline Young Investigators Award in Basic and Clinical Science 2009 & 6 & 4 & 67 \\
\hline Basic science in pediatric rheumatology & 5 & 0 & 0 \\
\hline Biomarkers in juvenile autoimmune diseases & 1 & 0 & 0 \\
\hline Complex issues in the management of back pain & 1 & 0 & 0 \\
\hline Cytokines and inflammatory mediators & 1 & 1 & 100 \\
\hline Headaches in pediatric rheumatology & 5 & 2 & 40 \\
\hline Imaging from cell to patient in pediatric rheumatology & 4 & 1 & 25 \\
\hline No child's play-Children and young people with rheumatic diseases & 4 & 0 & 0 \\
\hline Pediatric rheumatology & 153 & 72 & 47 \\
\hline Physiotherapy & 2 & 0 & 0 \\
\hline Rheumatism in young people & 2 & 0 & 0 \\
\hline Rheumatoid arthritis-anti-tumor necrosis factor therapy & 1 & 0 & 0 \\
\hline Spondyloarthropathies-Clinical aspects (other than treatment) & 1 & 1 & 100 \\
\hline Vasculitis: Clinical update and treatment & 1 & 1 & 100 \\
\hline "No place for Steroids": Novel therapeutic targets in systemic JIA & 3 & 0 & 0 \\
\hline "Too much and too little": Clinical challenges in pediatric rheumatology & 2 & 0 & 0 \\
\hline Total & 198 & 86 & 43.4 \\
\hline
\end{tabular}

We noted four abstracts having full-text publication date prior to the EULAR 2009 Congress and considered their publication time as zero. The elapsed date between the presentation in EULAR 2009 and the full text publication was median 19 months (range, 0 to 64 months).

The distribution of the abstracts and full-texts according to sub-categories, which appeared in EULAR Abstracts2View ${ }^{\mathrm{TM}}$ site, are given in the Table 1.

Juvenile idiopathic arthritis was the most studied disease in the abstracts $(n=112)$ and the full-texts $(\mathrm{n}=49)$, followed by systemic lupus erythematosus (10 abstracts and 5 full-texts). The disease types in the abstracts and full-texts are given in Table 2.

Italy (34 abstracts), United Kingdom (21 abstracts), Germany (20 abstracts), Turkey (14 abstracts), and Brazil (13 abstracts) were the top five countries according to abstract presentation. Italy (17 full-texts, 50\%), United Kingdom (10 full-texts, 48\%), Brazil (8 full-texts, 61.5\%), Turkey (8 full-texts, 57\%), and Germany (8 full-texts, 40\%) were also the top five countries according to full-text publication.

Even though EULAR is considered as an European Congress, following countries outside of the Europe (including Brazil which was among the

Table 2. Diseases in abstracts and full-texts

\begin{tabular}{|c|c|c|c|}
\hline & Abstract & Full-text & Abstract to full-text rate \\
\hline & $\mathrm{n}$ & $\mathrm{n}$ & $\%$ \\
\hline \multicolumn{4}{|l|}{ Disease } \\
\hline Juvenile idiopathic arthritis & 112 & 49 & 43.8 \\
\hline Systemic lupus erythematosus & 10 & 5 & 50 \\
\hline Periodical fever syndromes & 8 & 3 & 37.5 \\
\hline Familial Mediterranean fever & 7 & 1 & 14.3 \\
\hline Dermatomyositis & 7 & 3 & 42.9 \\
\hline Vasculitis & 5 & 4 & 80 \\
\hline Scleroderma & 5 & 1 & 20 \\
\hline Polyarteritis nodosa & 3 & 3 & 100 \\
\hline Henoch-Schönlein purpura & 2 & 1 & 50 \\
\hline Behçet's disease & 2 & 1 & 50 \\
\hline Others & 37 & 15 & 40.5 \\
\hline Total & 198 & 86 & 43.4 \\
\hline
\end{tabular}


top five countries) were found as contributors to EULAR 2009 in terms of pediatric rheumatology: Japan with seven abstracts (full-text ratio: 57\%), USA with six abstracts, Canada with three abstracts (full-text ratios: 100\%), Argentina with three abstracts (full-text ratio: 0\%), India, Saudi Arabia and Singapore with one abstract (full-text ratios: 100\%), and Morocco and Egypt with one abstract (full-text ratios: 0\%).

Thirty-five abstracts (17.7\%) were presented orally, while 163 abstracts (82.3\%) were presented as poster form. While 12 orally presented abstracts (34\%) became full-text articles, this rate was 45\% (74 abstracts) for poster presentations. There was no significant difference between abstract to full-text time according to presentation type as oral=19.5 months (range, 0 to 61 months) vs poster=19 months (range, 0 to 64 months) $(\mathrm{p}=0.832)$.
The Journal of Rheumatology (10 abstracts), Annals of the Rheumatic Diseases (nine abstracts), Clinical and Experimental Rheumatology (nine abstracts), Arthritis \& Rheumatology (seven abstracts), and Rheumatology (seven abstracts) were the journals in which most abstracts were published as full-text articles. Eighty-five of the full-texts (98.8\%) were published in a journal with an impact factor (five-year impact factor range, 1.237 to 54.390). The journal with the highest impact factor was The New England Journal of Medicine with a five-year impact factor of 54.390. Although the median impact factor of the journals published oral presentations as full-texts was higher than the journals published poster presentations as full-texts, the difference regarding the journals' impact factors between oral and poster presentations did not reach a statistically significant level, (4.592 vs 2.884, $\mathrm{p}=0.053$ ). Numbers of abstracts published as

Table 3. Journals where abstracts were published as full-texts

\begin{tabular}{lcc}
\hline & $\begin{array}{c}\text { Number of abstracts } \\
\text { published as } \\
\text { full-text articles }\end{array}$ & $\begin{array}{c}\text { Five-year impact } \\
\text { factor }\end{array}$ \\
\cline { 2 - 3 } \cline { 2 - 3 } & $\mathrm{n}$ & $\mathrm{n}$ \\
\hline The Journal of Rheumatology & 10 & 3.407 \\
Annals of the Rheumatic Diseases & 9 & 9.644 \\
Clinical and Experimental Rheumatology & 9 & 2.430 \\
Arthritis \& Rheumatology & 7 & 7.760 \\
Rheumatology (Oxford, England) & 7 & 4.592 \\
Pediatric Rheumatology Online Journal & 6 & 1.710 \\
Arthritis Care \& Research & 4 & 4.962 \\
Lupus & 3 & 2.331 \\
Modern Rheumatology & 3 & 2.086 \\
Rheumatology International & 3 & 1.545 \\
Clinical Rheumatology & 2 & 1.913 \\
The Journal of Pediatrics & 2 & 4.152 \\
The British Journal of Radiology & 2 & 1.976 \\
Journal of Proteome Research & 1 & 4.481 \\
Genes and Immunity & 1 & 3.113 \\
Pharmacogenetics and Genomics & 1 & 3.561 \\
Blood & 1 & 9.567 \\
Annals of the New York Academy of Sciences & 1 & 3.838 \\
Ugeskrift for Laeger & 1 & NA \\
JAMA & 1 & 31.026 \\
The New England Journal of Medicine & 1 & 54.390 \\
Arthritis Research and Therapy & 1 & 4.658 \\
Joint, Bone, Spine & 1 & 2.557 \\
Scandinavian Journal of Rheumatology & 1 & 2.391 \\
Jornal de Pediatria & 1 & 1.237 \\
Molecular Biology Reports & 1.908 \\
Pediatric Radiology & 1 & 1.591 \\
Clinics (Sao Paulo, Brazil) & 1 & 1.368 \\
Acta Radiologica & 1.645 \\
International Journal of Rheumatic Diseases & 1 & 1.705 \\
Journal of Pediatric Gastroenterology and Nutrition & 2.758 \\
Pediatric Allergy and Immunology & 1 & 2.884 \\
\hline
\end{tabular}


full-texts in each journal are shown in Table 3. Sixty-three (73.3\%) full-texts were published as full-texts in a rheumatology journal, while other journals were related to the fields of pediatrics, genetics, general medicine, and radiology.

\section{DISCUSSION}

This study aimed to investigate the publication rates of the abstracts presented in the EULAR 2009 that were related to pediatric rheumatology. Our results showed that approximately three out of five abstracts (43.4\%) were published in the full-text form in 19 months, in a duration of approximately five years (64 months). This data conform with those reported previously (Table 4).

It was advocated that full text publication rates after a congress indicate the scientific value of the congress. ${ }^{4}$ Scherer et al. ${ }^{10}$ reported full text publication rates of medical abstracts as $44.5 \%$ in their Cochrane review. We found that the publication rate of pediatric rheumatology related abstracts in EULAR 2009 almost reached this rate. Therefore, it can be suggested that even though EULAR is a general rheumatology congress, it is a beneficial meeting for all attendees engaged in pediatric rheumatology.

However, Yilmaz et al. ${ }^{11}$ found the publication rate of all abstracts presented in EULAR 2008 as $34.7 \%$. According to our results (43.4\%), publication rate of abstracts related to pediatric rheumatology was higher than general full-text publication rates of EULAR. Several reasons can be accounted for this difference. First, Yilmaz et al. ${ }^{11}$ conducted their investigations three years after the congress, while we evaluated a five-year period after the congress. This difference in time may have caused the difference in publication rates. Second, their study included all oral and poster abstracts and therefore studies with less chance of publication could be included into the analysis. It was reported that the publication rate of allied health topic abstracts such as psychology, education, and physical and occupational therapy was only around $10 \% .^{3}$

The rates reported in the present study seem higher than the first report of Hashkes et $\mathrm{al}^{3}{ }^{3}$ about the publication rates of a pediatric rheumatology meeting. In the mentioned study, the authors investigated the abstracts of the

Table 4. Rates of abstract publications from present study and other rheumatology meetings

\begin{tabular}{|c|c|c|c|c|}
\hline \multirow[t]{2}{*}{ Meetings } & \multirow{2}{*}{$\begin{array}{c}\text { Abstracts published } \\
\%\end{array}$} & \multicolumn{2}{|c|}{$\begin{array}{l}\text { Elapsed months until } \\
\text { full-text publication }\end{array}$} & \multirow[b]{2}{*}{$p$} \\
\hline & & Median & Min.-Max. & \\
\hline \multicolumn{5}{|l|}{ Present study } \\
\hline \multicolumn{5}{|c|}{ EULAR 2009} \\
\hline \multicolumn{5}{|c|}{ (Pediatric Rheumatology Related Abstracts) } \\
\hline Oral & 34 & 19.5 & $0-61$ & \\
\hline Poster & 45 & 19 & $0-64$ & \\
\hline Overall & 43.4 & 19 & $0-64$ & \\
\hline Oral versus poster & & & & 0.832 \\
\hline \multirow{2}{*}{\multicolumn{5}{|c|}{$\begin{array}{l}\text { Other Rheumatology Meetings } \\
4^{\text {th }} \text { Park City Pediatric Rheumatology Meeting } 1998 \\
\text { (All Abstracts) }\end{array}$}} \\
\hline & & & & \\
\hline Overall & 36 & 24 & $0-48$ & \\
\hline \multirow{2}{*}{\multicolumn{5}{|c|}{$\begin{array}{l}\text { ACR/ARHP } 2006 \\
\text { (All Abstracts) }^{12}\end{array}$}} \\
\hline \multicolumn{4}{|l|}{$(\text { All Abstracts })^{12}$} & \\
\hline Oral & 68.5 & 14.1 & $0-61$ & \\
\hline Poster & 57.1 & 15.4 & $0-61$ & \\
\hline Overall & 59.1 & 18.2 & $0-61$ & \\
\hline Oral versus poster & & & & NA \\
\hline \multicolumn{5}{|l|}{ EULAR 2008} \\
\hline \multicolumn{5}{|c|}{ (All Oral and Poster Abstracts) $)^{11}$} \\
\hline Oral & 44.2 & & & NA \\
\hline Poster & 33 & & & NA \\
\hline Overall & 34.7 & 13 & $0-31$ & \\
\hline Oral versus poster & & & & NA \\
\hline
\end{tabular}


$4^{\text {th }}$ Park City Pediatric Rheumatology Meeting, which was held in 1998 in USA, and reported a publication rate of $36 \%$. Over a four-year period after the meeting, the authors reported a median publication time of two years, which is longer than the results obtained in this study. In the light of the present results, we may suggest that the productivity of pediatric rheumatology has been increasing since then. This may be related to the increasing number of rheumatology journals where authors can find place for their manuscripts and increase the overall quality of work.

On the other hand, the elapsed time until publication in our study was longer than the other reported publication rates of rheumatology related meetings. ${ }^{11,12}$ This may be attributed to the specificity of the topic. As pediatric rheumatology is a subarea of the rheumatology field, the quota for pediatric rheumatology per issue might be limited in journals, which may explain the related time lag.

Being the most common disease in pediatric rheumatology, juvenile idiopathic arthritis was the most studied disease in EULAR 2009. Juvenile idiopathic arthritis was also the most studied disease in the previous report of Hashkes et al., ${ }^{3}$ while systemic lupus erythematosus ranked in the second place both in Hashkes' and our studies.

It was reported before that presentation type might have an impact on full-text publication rate and oral presentations might be favorable in the process of full-text publication. ${ }^{12}$ However, in the present study, publication rate was higher in the poster presentations ( $45 \%$ vs $34 \%$ ). Most of the abstracts $(82.3 \%)$ were presented in the poster form in EULAR 2009 and this may have had an effect on full-text publication rate. Also, there were some orally presented abstracts, such as patient experiences or project advertisements, which were not intended for a full-text publication.

We detected no difference between presentation types in terms of elapsed times until presentation and the impact factors of published journals. In terms of journals' impact factors, we found a $p$ value of 0.053 between oral and poster presentations, which may be interpreted as a significant difference. In this case, we may conclude that oral presentations could be published in journals with higher impact factors, since the median impact factor of journals where oral presentations were published was higher (oral: 4.592 vs poster: 2.884 ). This result is in concordance with the previous reports. ${ }^{12}$

The first journal where the highest number of full-texts were published was the Journal of Rheumatology, as Hashkes et al. ${ }^{3}$ reported. Although the official journal of EULAR is Annals of the Rheumatic Diseases, this journal ranked in second place according to publication rates following the Journal of Rheumatology. In the previous reports of adult rheumatology congresses, Arthritis and Rheumatism (it has been divided into two independent journals as Arthritis \& Rheumatology and Arthritis Care \& Research) ranked in first place in terms of publication rates. ${ }^{11,12}$

Even though EULAR is a European meeting for the rheumatology field, it was interesting to see many studies from countries outside of Europe presented in EULAR at least in the field of pediatric rheumatology.

We hope that the data obtained in our study may assist in coming to conclusions about the progression of pediatric rheumatology by allowing a comparison between our results and those previously reported. While the strength of our study was the systematic search used, it is likely that our publication rates were higher due to publication at local journals.

Searching the full-texts in the journals using in PubMed, Web of Science and Google Scholar could be counted as a limitation, due to not all the journals were indexed in these databases. This could affect the results.

In conclusion, it seems that even though EULAR is a general rheumatology congress, it has an important place in pediatric rheumatology field as well. EULAR is an opportunity for pediatric rheumatologists as a useful platform to meet and share ideas.

\section{Declaration of conflicting interests}

The authors declared no conflicts of interest with respect to the authorship and/or publication of this article.

\section{Funding}

The authors received no financial support for the research and/or authorship of this article. 


\section{REFERENCES}

1. Schaller JG. The history of pediatric rheumatology. Pediatr Res 2005;58:997-1007.

2. Balasubramanian SP, Kumar ID, Wyld L, Reed MW. Publication of surgical abstracts in full text: a retrospective cohort study. Ann R Coll Surg Engl 2006;88:57-61.

3. Hashkes P, Uziel Y. The publication rate of abstracts from the 4th Park City Pediatric Rheumatology meeting in peer-reviewed journals: what factors influenced publication? J Rheumatol 2003;30:597-602.

4. Smith HD, Bogenschutz ED, Bayliss AJ, Altenburger PA, Warden SJ. Full-text publication of abstractpresented work in physical therapy: do therapists publish what they preach? Phys Ther 2011;91:234-45.

5. Available from: http://www.abstracts2view.com/ eular/ [Accessed: December 16, 2015]

6. Available from: http://www.ncbi.nlm.nih.gov/pubmed [Accessed: December 16, 2015]
7. Available from: http://apps.webofknowledge.com/ [Accessed December: 16, 2015]

8. Available from: http://scholar.google.com.tr/ [Accessed: December: 16, 2015]

9. Available from: http://admin-apps.webofknowledge. com/JCR/JCR [Accessed: December 16, 2015]

10. Scherer RW, Langenberg $P$, von Elm E. Full publication of results initially presented in abstracts. Cochrane Database Syst Rev 2007;2:MR000005.

11. Yilmaz S, Kalyoncu U, Cinar M, Karadag O, Koca SS, Simsek I, et al. Features and publication rates of scientific abstracts presented at a rheumatology congress--EULAR 2008. Bull Hosp Jt Dis (2013) 2013;71:124-7.

12. Amarilyo G, Woo JM, Furst DE, Hoffman OL, Eyal $\mathrm{R}$, Piao C, et al. Publication outcomes of abstracts presented at an American College of Rheumatology/ Association of Rheumatology Health Professionals annual scientific meeting. Arthritis Care Res (Hoboken) 2013;65:622-9. 